\title{
THE INVESTIGATION OF STRUCTURE HETEROGENEOUS JOINT WELDS IN PIPELINES
}

\author{
Lyudmila Lyubimova ${ }^{1}$, Roman Fisenko ${ }^{1}$, Sergei Breus ${ }^{1}$, and Roman Tabakaev ${ }^{1, *}$ \\ ${ }^{1}$ National Research Tomsk Polytechnic University, 634050 Tomsk, Russia
}

\begin{abstract}
Welding joints of dissimilar steels don't withstand design life. One of the important causes of premature destructions can be the acceleration of steel structural degradation due to cyclic mechanical and thermal gradients. Two zones of tube from steel 12H18N9T, exhibiting the structural instability at early stages of the decomposition of a supersaturated solid austenite solution, were subjected to investigation. Methods of x-ray spectral and structure analysis, micro hardnessmetry were applied for the research. Made the following conclusions, inside and outside tube wall surfaces of hazardous zones in welding joint have different technological and resource characteristics. The microhardness very sensitive to changes of metal structure and can be regarded as integral characteristic of strength and ductility. The welding processes are responsible for the further fibering of tube wall structure, they impact to the characteristics of hot-resistance and long-term strength due to development of ring cracks in the welding joint of pipeline. The monitoring of microhardness and structural phase conversions can be used for control by changes of mechanical properties in result of post welding and reductive heat treatment of welding joints.
\end{abstract}

\section{Introduction}

The main structural materials for the manufacture of superheaters for boiler, working on supercritical pressure, in Russia, including the welding joints, are austenitic steels $12 \mathrm{H} 18 \mathrm{~N} 9 \mathrm{~T}$ (Russian designation is $12 \mathrm{X} 18 \mathrm{H} 9 \mathrm{~T}$ ) and pearlitic steels 12H1MF (Russian designation is $12 \mathrm{X} 1 \mathrm{MF}$ ). Calculated on resource of work about 300 thousand hours, they are destroyed much earlier due to various reasons $[1,2]$. The typical state for welding joints is the presence of chemical, structural, mechanical and geometrical heterogeneities, which are strain concentrators [3]. One of the important causes of premature destructions can be the acceleration of steel structural degradation due to cyclic mechanical and thermal gradients [4-6]. The structural conversions have a particular negative effect on welding joints resistance to brittle failure. The technical diagnostic of welding joints, basis on specialized scientific and technical documents, involves investigation of metal composition, structure and properties.

\footnotetext{
*Corresponding author: TabakaevRB@tpu.ru
} 
In this regard, a material structure investigation in the hazardous areas of welding joint is remained actual task.

\section{Material and methods}

Methods of X-ray spectral analysis with using of x-ray fluorescence analyzer EDX 2800 (SKYRAY, USA), x-ray structure analyzer DRON-3 (Russia) and microhardnessmetry with using of microhardness tester PMT-3 (Russia) were applied for the research.

Two zones of tube from steel $12 \mathrm{H} 18$ N9T (fig. 1), exhibiting the structural instability at early stages of the decomposition of a supersaturated solid austenite solution, were subjected to investigation [7]. Zone No.1 is located at a distance $7.5 \mathrm{~mm}$ from axis of welding joint, zone No.2 is located at a distance $14.5 \mathrm{~mm}$. Zone No.1 called heat-affected zone (HAZ), No.2 - a zone of thermal welding influence (in accordance with hot-tinting).

a)

b)

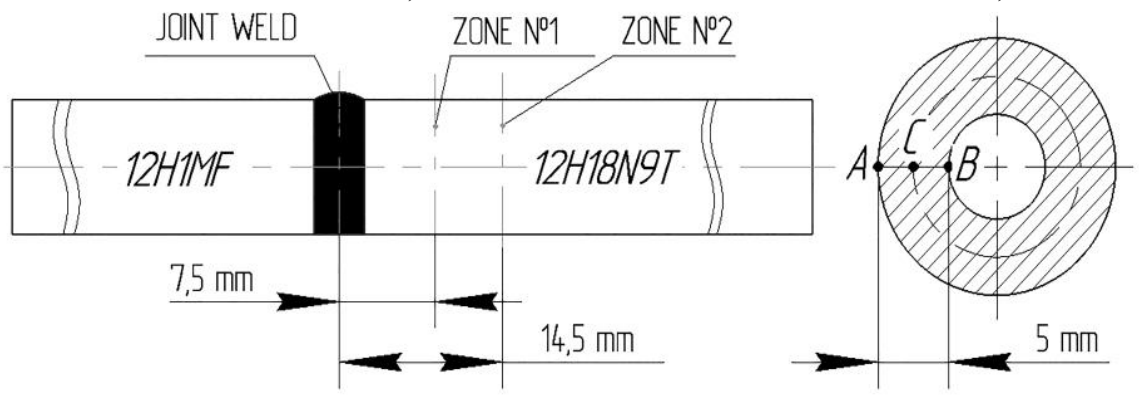

Fig. 1. The scheme of welding joint: a) zones No.1 and No.2 for investigation; b) AC, BC - the directions of microhardness measurements at tube wall thickness.

\section{Results and discussion}

The experimental results are shown in figures 2 and 3.

From figure 2 implies that along of thermal influence of welding take place a heterogeneous decomposition of the supersaturated solid austenite solution, which reveals itself by the phenomena of structural polymorphism, in result of both samples is acquired a mixed phase structure. One phase has a body-cented cubic (BCC) lattice, second phase is austenite $(\gamma-\mathrm{Fe})$ with face-centered cubic (FCC) lattice. For zone No.1 the concentration of $\alpha$-Fe and $\gamma$-Fe on outside surface are accordingly 81 and 19 mas $\%$, for inside surface -83 and $16 \%$. For zone No.2 the concentration of $\alpha$ - and $\gamma$-phases on outside surface are accordingly 89 and $11 \%$, inside surface is presented only $\alpha$-phase of Fe.

The result of heterogeneous solid solution decomposition is the emergence of point defects in kind of exempt substitutional impurities and their inhomogeneous distribution (fig. 3a, b). The process of allocating is reversible and accompanied by dissolving of impurities at presence of temperature and strain gradients. This is significantly different processes, because dissolution is accompanied by the formation of vacancy porosity on the dissolution front, void coalescence and formation of microcracks. The final stage of this defect is macrocrack. 
a)

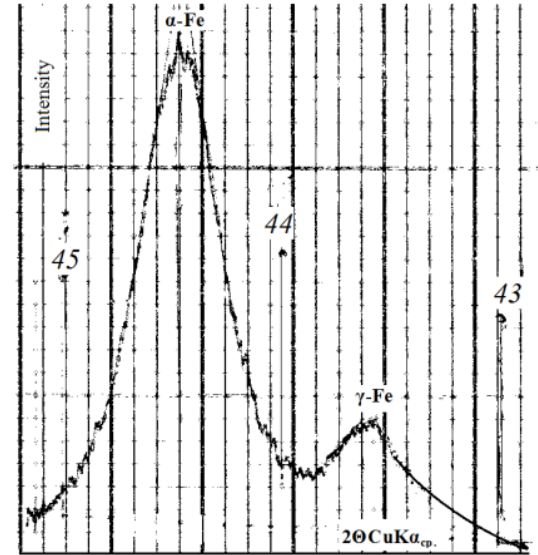

Outside surface

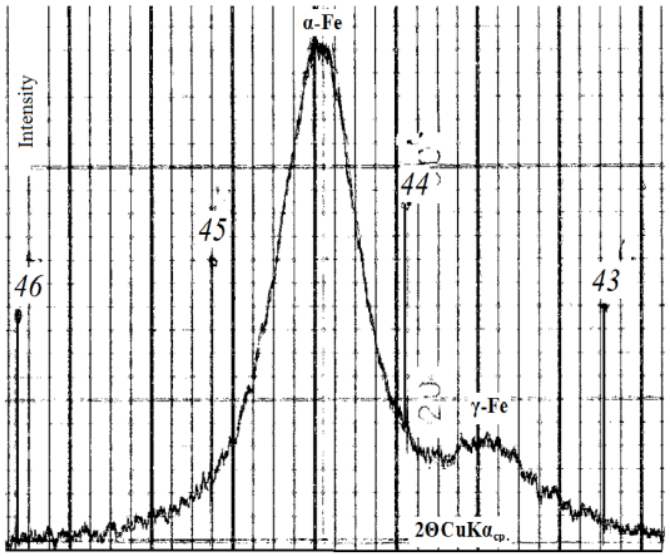

Inside surface

b)

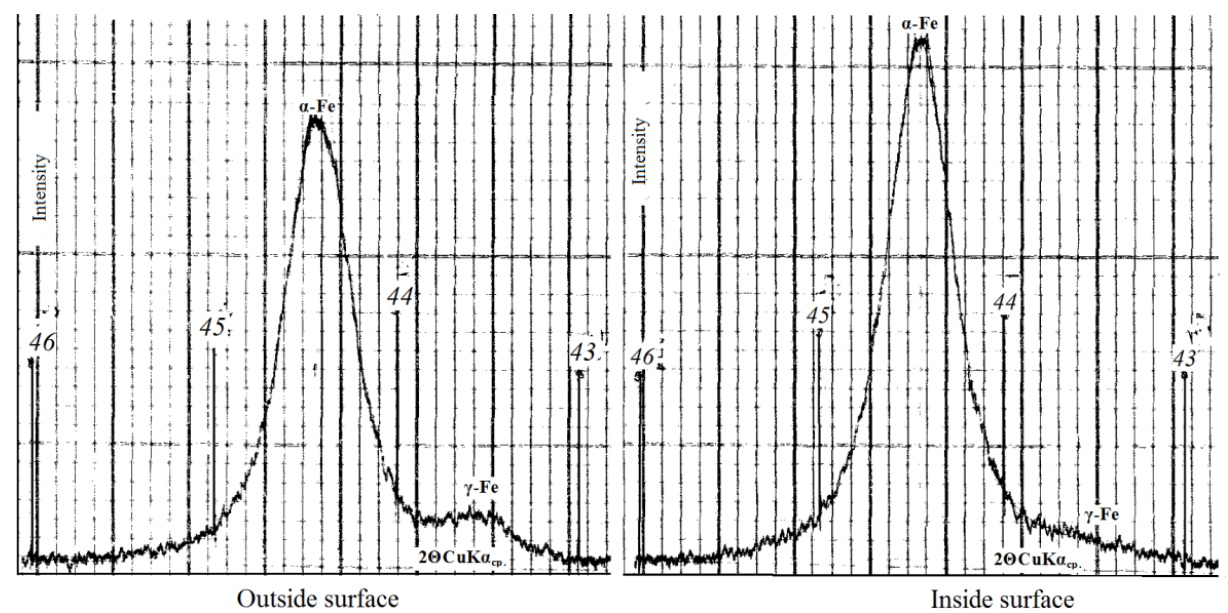

Fig. 2. Fragments of radiographs: a) sample from zone No.1; b) sample from zone No.2.

It is known [ 8 and others], that $80-85 \%$ forced stops of thermal power plants boilers is due to damage of tube heating surfaces. The tubes, from which produced heating surfaces, in supply condition due to difficult technological chain of their processing (drawing, rolling, annealing and etc.) is acquired the anisotropy of properties, caused by heterogeneity and discontinuity of structure that provides the spread in values of mechanical characteristics along the tube length and wall thickness. To confirm of this position in present work is measured the microhardness along wall thickness of superheater tube from heat-affected zone and zone of thermal welding influence of welding joint from heterogeneous steels $12 \mathrm{H} 1 \mathrm{MF}$ and 12H18N9T (in accordance with fig. 1), results of which are shown in fig. 4.

Figure 4 is shown that the parameters of microhardness, and also its distribution, are different at measurement from outside and inside surfaces to centre of tube wall (in accordance with fig. 1).

The microhardness distributions along wall thickness are revealed its fibering by levels of microhardness. For example, in heat-affected zone microhardness at outside surface is $1700 \mathrm{MPa}$, at inside - $3400 \mathrm{MPa}$, at the middle of wall - $2500 \mathrm{MPa}$. The maximum $4000 \mathrm{MPa}$ and the minimum of microhardness $1000 \mathrm{MPa}$ are reached near the middle of wall. Inside of these ranges microhardness is strongly oscillated (fig. 4a). 
a)

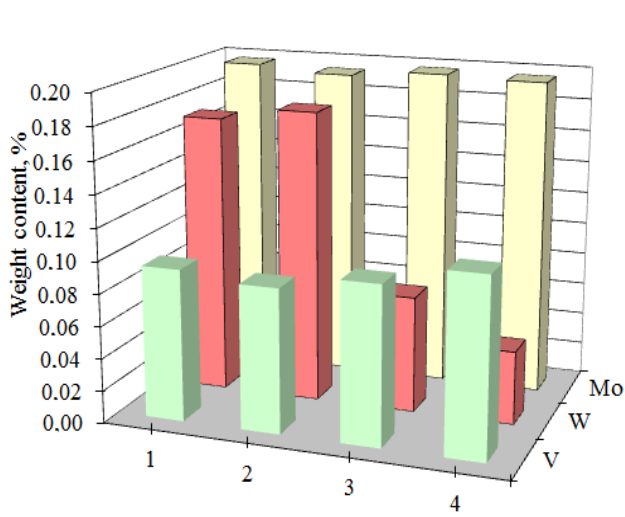

c)

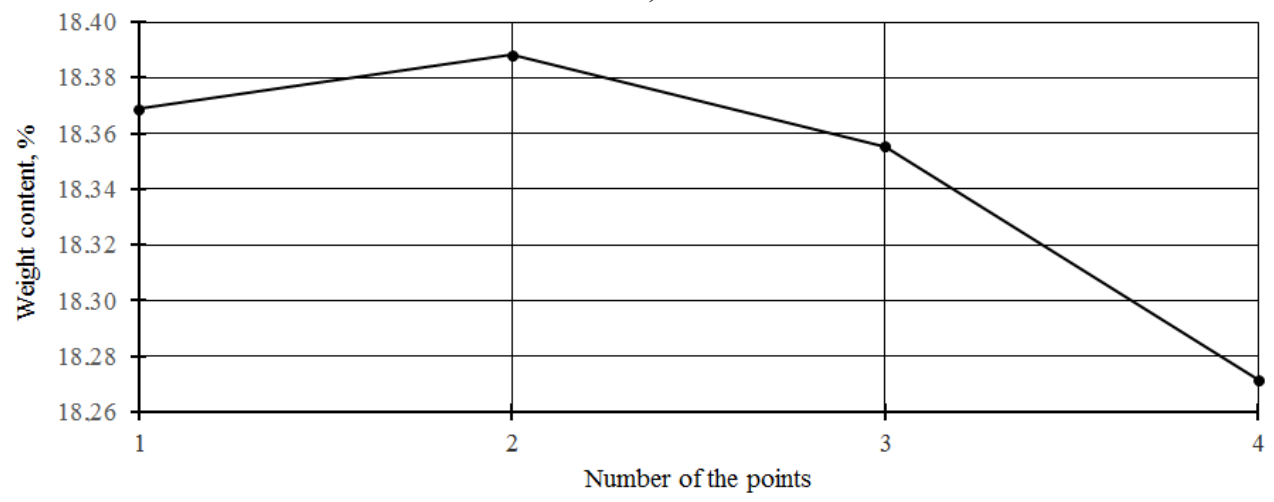

b)

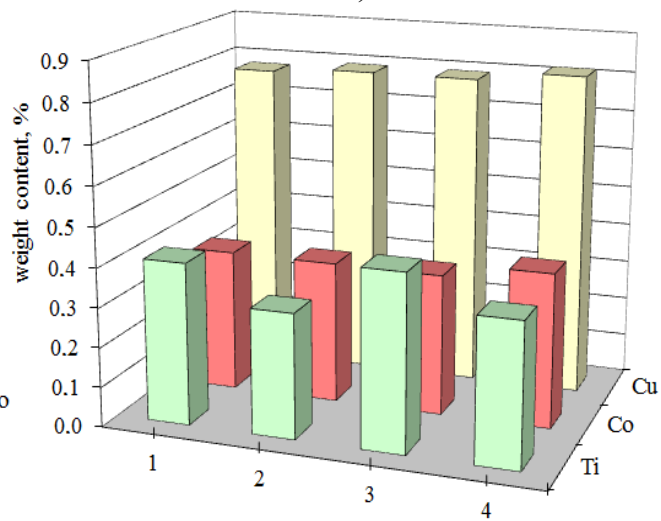

Fig. 3. The results of spectral analysis: a) the concentration of alloying elements $V, W, M o$; b) the concentration of alloying elements $\mathrm{Ti}, \mathrm{Co}, \mathrm{Cu}$; c) the concentration of chrome (points 1, 3-inside surface of zones No.1 and No.2 accordingly; points 2, 4 - outside surface of zones No.1 and No.2).

In zone of thermal welding influence microhardness distribute thus: the maximum $3500 \mathrm{MPa}$ is detected near the middle of tube wall, at which the microhardness decreases to outside and inside surfaces to a value about $1700 \mathrm{MPa}$. In this case microhardness also oscillated from 1400 to $3100 \mathrm{MPa}$ (fig. 4b).

The microhardness is parameter of strength, which characterize a resistance of material to deformation and destruction [9]. The presence of significant spread in microhardness values provides spread of strength metal properties and, consequently, resource of heating spirals, working in the same conditions.

Presented in fig. 4 zonal microhardness distribution along wall thickness lead to decrease of hot-resistance in conditions of long-term operation due to emergence and development of ring cracks in the welding joint of pipeline.

This conclusion is confirmed by detected lamination of superheater tube wall (fig. 5) in result of deep oxidation at high temperature. 
a)

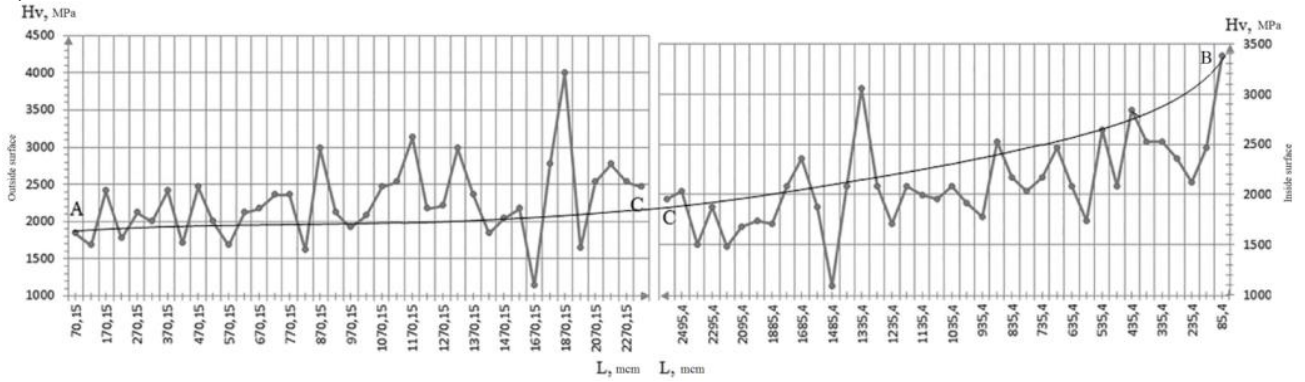

b)

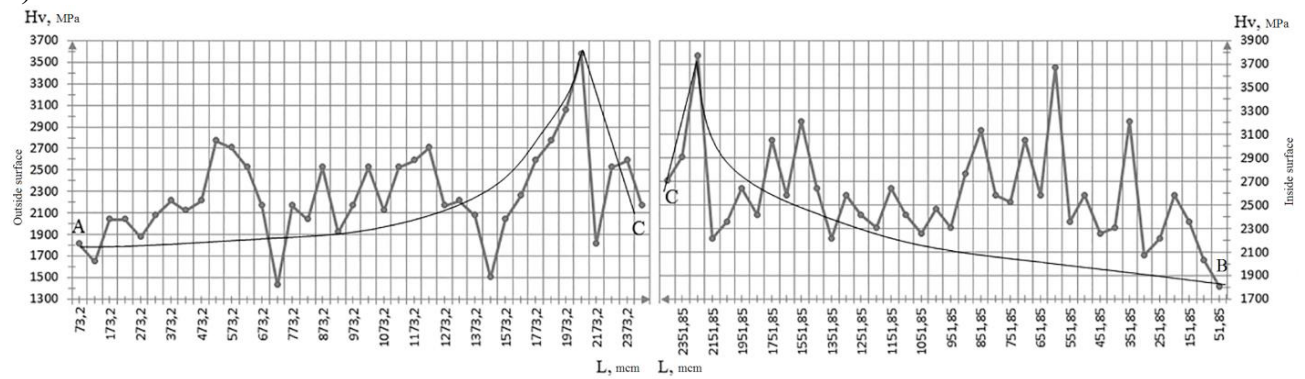

Fig. 4. The microhardness along wall thickness of welded tube: a) zone, located at a distance $7.5 \mathrm{~mm}$ from axis of the welding joint; $b$ ) zone, located at a distance $14.5 \mathrm{~mm}$ from axis of the welding joint; $\mathrm{AC}, \mathrm{BC}-$ the directions of microhardness measurements at tube wall thickness (in accordance with fig. 1).

a)

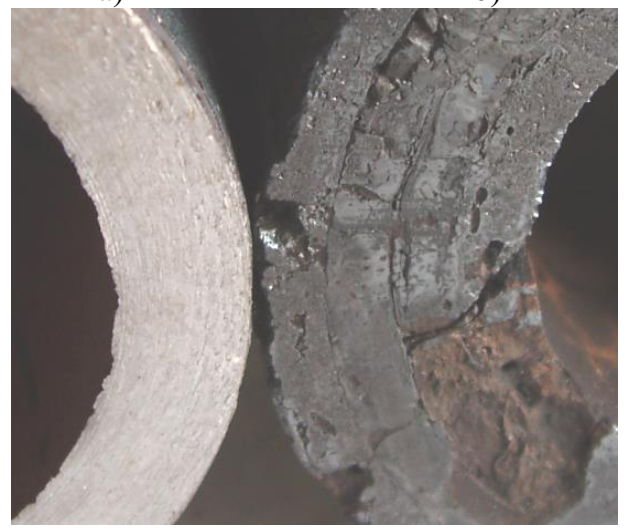

c)

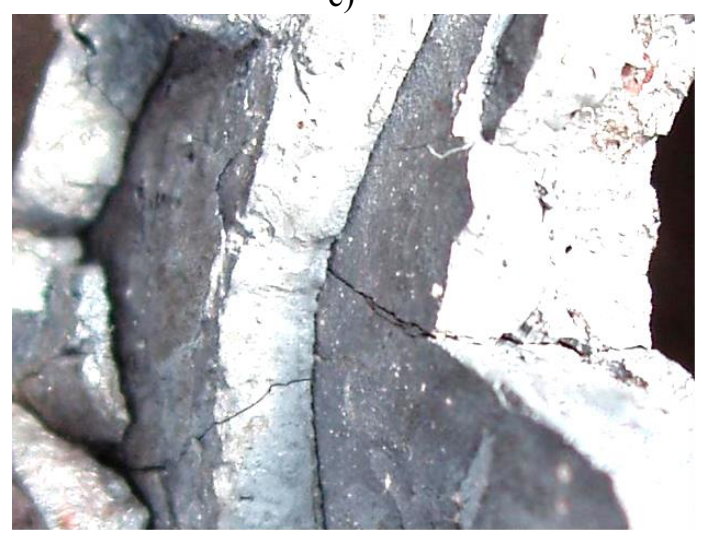

Fig. 5. The layered tube structure: a) the pipe wall in the initial state; b, c) the pipe wall after different stages of deep oxidation.

\section{Conclusions}

The results of completed investigation are follows:

1. Inside and outside tube wall surfaces of hazardous zones in welding joint have different technological and resource characteristics.

2. The microhardness very sensitive to changes of metal structure. During welding, heating, mechanical loads the microhardness changes in direction of yield strength change 
and, as the hardness of separate micro-local areas of material microstructure, is a very difficult property, which can be regarded as integral characteristic of strength and ductility.

3. The welding processes are responsible for the further fibering of tube wall structure, creating zones with strongly different strength properties, reducing the resistance to destruction and impacting to the characteristics of hot-resistance and long-term strength due to development of ring cracks in the welding joint of pipeline.

4. The monitoring of microhardness and structural phase conversions can be used for control by changes of mechanical properties in result of post welding and reductive heat treatment of welding joints.

\section{Acknowledgments}

The reported study was funded by RFBR according to the research project No. 15-0899544 A.

\section{References}

1. V.A. Bogachev, G.A. Urusova, M.A. Shumovskaya, Pow. Tech. Eng. 48, 467 (2015)

2. V.A. Bogachev, D.S. Zmienko, A.E. Korneev, T.P. Pshechenkova, B.E. Shkol'nikova, Therm. Eng. 59, 274 (2012)

3. J.S. Lee, K. Maruyama, Met. Mat. Int. 21, 639 (2015)

4. L.L. Lyubimova, A.A. Makeev, A.A. Tashlykov, A.S. Zavorin, R.N. Fisenko, Steel in Transl. 44, 117 (2014)

5. L.L. Lyubimova, A.A. Makeev, A.S. Zavorin, A.A. Tashlykov, A.I. Artamontsev, B.V. Lebedev, R.N. Fisenko, Therm. Eng. 61, 600 (2014)

6. L. Lyubimova, R. Tabakaev, A. Tashlykov, A. Zavorin, V. Zyubanov, EPJ Web Conf. $110(2016)$

7. A.A. Makeev, L.L. Lyubimova, A.S. Zavorin, A.A. Tashlykov, Steel in Transl. 39 (2009)

8. E.Y. Priymak, V.I. Gryzunov, Met. Sc. Heat Treatm. 53, 136 (2011)

9. Y. Jin, R. Li, Z. Yu, Y. Wang, J. Mat. Eng. Perf. 25, 1327 (2016) 